\title{
Residual bioburden in reprocessed side-view endoscopes used for endoscopic retrograde cholangiopancreatography (ERCP)
}

Authors

Institutions
D. L. N. L. Ubhayawardana' ${ }^{1}$, J. Kottahachchi' ${ }^{1}$, M. M. Weerasekera' ${ }^{1}$ I. W. M. P. Wanigasooriya ${ }^{2}$, S. S. N. Fernando ${ }^{1}$, M. De Silva²

${ }^{1}$ Department of Microbiology, Faculty of Medical Sciences, University of Sri Jayewardenepura, Nugegoda, Sri Lanka

${ }^{2}$ Endo-Therapy Unit, Colombo South Teaching Hospital, Kalubowila, Sri Lanka

\section{Bibliography}

Dol http://dx.doi.org/

10.1055/s-0033-1359234

Endoscopy International Open

2013; 1: E12-E16

(c) Georg Thieme Verlag KG

Stuttgart · New York

E-ISSN 2196-9736

Corresponding author:

J. Kottahachchi

Department of Microbiology,

University of Sri

Jayewardenepura,

Gangodawila, Nugegoda

Western 10250

Sri Lanka

jananiekottahachchi@yahoo.

com

fneluka@gmail.com
Background and study aim: Worldwide some endoscopy units routinely continue to use manual reprocessing techniques for disinfection of sideview endoscopes. The aim of this study was to evaluate the outcome quality of manual reprocessing techniques for removal and inactivation of the bioburden from side-view endoscopes used for endoscopic retrograde cholangiopancreatography (ERCP) in a tertiary referral endotherapy unit in Sri Lanka.

Methods: 102 samples obtained from two different flexible side-view endoscopes (Olympus TJF Q $180 \mathrm{~V}$ and Olympus TJF 160 R) were tested for microbial growth. Three samples were collected each time; one swab from the tip before and another after manual reprocessing. The third sample

\section{Introduction \\ $\nabla$}

Endoscopic retrograde cholangiopancreatography (ERCP) is well established as a technique for minimal access endotherapy of biliary and pancreatic diseases. In contrast to other gastrointestinal endoscopic procedures, ERCP is somewhat vulnerable to transmission of infections [1]. The side-view endoscope is more difficult to clean and disinfect because of its side-view orientation, small lumen, multiple channels and the length of insertion tube $[2,3]$. Flexible gastrointestinal endoscopes show a high rate of microorganisms after use (from $10^{5}$ colony-forming units [CFU]/ $\mathrm{ml}$ to $10^{10} \mathrm{CFU} / \mathrm{ml}$ ) [4-7]. This is especially found when time-consuming therapeutic processes are performed in the potentially infected biliary and pancreatic tract [8]. Endoscopes are reusable instruments, employed in different patients in a single day, and appropriate reprocessing of endoscopes and accessories is mandatory prior to each individual procedure $[3,9]$.

The incidence of transmission of infections through flexible endoscopes is very low and has been found to be 1 in 1.8 million procedures [10]. was collected by flushing the working channel with sterile normal saline after manual reprocessing. Microorganisms were identified by culturing the samples.

Result:: After reprocessing, culture-positive rates were $20 \%$ and $9 \%$ for the samples obtained from the tip and the working channel of the side-view endoscopes, respectively. Klebsiella spp. and Candida spp. were found to be the commonest microorganisms in the samples from the tips and from the working channels, respectively, of the reprocessed side-view endoscopes.

Conclusion: There is a high culture-positive rate after reprocessing of the side-view endoscopes using the manual reprocessing procedure, despite strict adherence to the protocol for reprocessing.

However, endoscopes have been associated with outbreaks of nosocomial infections [11]. Since most of the studies were based on culture identification methods, the true bioburden may have been underestimated because of the likely presence uncultivable microorganisms and because of biofilm formation. The infection rate may have also been underestimated because of factors such as incomplete surveillance; underreporting, and asymptomatic infections $[12,13]$. Hence although the risk of endoscopy-related infection is documented to be very low, continued efforts are needed to ensure that quality is maintained during endoscope reprocessing to reduce the incidence of endoscopy-related infections.

A number of studies have highlighted that pathogen transmission related to endoscopy is due to failure to follow established cleaning and disinfection/sterilization guidelines or due to the use of defective equipment $[12,14,15]$. In addition, improper intravenous administration of anesthetics during gastrointestinal endoscopy has been found to be another common mode of transmission of pathogens [9]. Although the Centers for Disease Control and Prevention (CDC) have not 
recommended routine microbiological surveillance of gastrointestinal endoscopes [16], monitoring of the outcome quality of endoscope reprocessing methods is recommended by the European Societies of Gastrointestinal Endoscopy (ESGE) and of Gastroenterology Endoscopy Nurses and Associates (ESGENA) [17]. The purpose of this study was to evaluate the outcome quality of the manual reprocessing procedure for removal and inactivation of the bioburden from the side-view endoscopes in a tertiary referral endotherapy unit in Sri Lanka.

\section{Materials and methods}

\section{$\nabla$}

The study was carried out from September 2012 to February 2013 at the endotherapy unit of the Colombo South Teaching Hospital and the Department of Microbiology of the University of Sri Jayewardenepura, Sri Lanka.

A total of 102 samples were collected from two different sideview endoscopes (Olympus TJF Q 180V, Olympus TJF 160R) that had been used for both diagnostic and therapeutic purposes, regardless of the underlying disease of the patient.

\section{Sampling for microbiological cultures}

The tip and the working channel of the side-view endoscopes were monitored as these two sites have been shown to have a high bioburden after ERCP [18]. The sampling method, using normal saline and swabs, followed the ESGE-ESGENA guideline for quality assurance in reprocessing: Microbiological surveillance testing in endoscopy [17]. Swabbing is the ideal sampling method for the tip; this is supported by other publications.

Three types of sample were collected from each endoscope. The first sample was taken (in duplicate) from the tip (including the elevator forceps) of the insertion tube, using a sterile swab soaked with sterile normal saline immediately after withdrawal of the endoscope following ERCP. The second swab samples were taken (in duplicate) after the protocol-based manual reprocessing of the side-view endoscope had been completely performed. The third sample was obtained after manual reprocessing by collecting $10 \mathrm{ml}$ of sterile normal saline through the working channel. Sterile saline was injected into the working channel via biopsy valve using a sterile syringe, and subsequently collected at the distal tip of the side-view endoscope. Aseptic techniques were applied during the collection.

The specimens were transported to the bacteriology laboratory at the Department of Microbiology, Faculty of Medical Sciences, University of Sri Jayewardenepura within 2 hours of collection and were subsequently processed.

\section{Culturing of samples}

One of the two swabs taken before and after the procedures was directly inoculated onto blood agar and MacConkey agar plates. The remaining duplicate swabs taken before and after the procedure were enriched in brain heart infusion (BHI) broth. The plates and the broth were incubated for 18 hours at $37^{\circ} \mathrm{C}$. The BHI broths were then inoculated on blood agar and MacConkey agar plates irrespective of the turbidity. The normal saline samples were centrifuged at $1500 \mathrm{~g}$ for 10 minutes. One drop $(0.001 \mathrm{ml})$ was taken from the sediment, inoculated on solid media as above, and incubated to obtain isolated colonies [11]. If no growth was seen, plates were incubated for another 24 hours.

Culture results were reported as positive or negative for all the swab samples regardless of the number of colonies. In acccordance with the ESGE-ESGENA guideline [17], microbial growth with $>20 \mathrm{CFU} / \mathrm{channel}$ was considered to be significant growth for the normal saline samples taken from the side-view endoscopes after reprocessing. Isolated microorganisms were identified according to standard determination schemes.

\section{Manual eprocessing of side-view endoscopes}

In the endotherapy unit at Colombo South Teaching Hospital reprocessing of the side-view endoscopes is done in accordance with the manufacturer's instructions. Manual reprocessing is performed by trained nurses with more than 1 year's experience in this procedure. As soon as the ERCP procedure is over, the outer surface of the insertion tube is wiped using a piece of gauze soaked with a detergent. The working channel is then flushed by immersing the tip of the scope in the detergent solution and suctioning the detergent solution through the channnel.

The side-view endoscope is then moved to reprocessing basin (Manual Disinfector TD 20, Olympus) where manual cleaning is performed by washing all debris from the exterior. All removable parts are separately cleaned. Then, using an all-channel irrigator, the channels of the scope are flushed with water. The side-view endoscope and the accessories used are then rinsed in $0.35 \%$ peracetic acid for 10 minutes in a closed system [16]. The scope is then submerged in filtered water to flush all channels with filtered water. Finally, the ready-to-use scope is dried using a sterile piece of gauze. The total time required to carry out manual reprocessing of the side-view endoscope is about 18 minutes. At the end of the endoscopy clinic, in addition to the manual reprocessing described above, $70 \%$ isopropyl alcohol is flushed though the internal channels and allowed to air-dry.

\section{Results}

$\nabla$

The side-view endoscopes were sampled on 102 occasions, 67 from the Olympus TJF Q $180 \mathrm{~V}$ and 35 from the Olympus TJF $160 \mathrm{R}$. The results of the microbial cultures from the 6 -month period are summarized in - Table 1. After completion of reprocessing, the culture-positive rate for the tips of the two side-view endoscopes was $20 \%(21 / 102)$ and that for the working channels was $9 \%$ (10/102). Out of the 21 culture-positive samples taken from the tip of the reprocessed side-view endoscopes $71 \%$ (15/21) were from the 1-year-old side-view endoscope (Olympus TJF Q 180V) while $29 \%(6 / 21)$ were from the 5-year-old side-view endoscope

Table 1 Sampling and culture results for the tip (before and after reprocessing) and the working channel (after reprocessing) of the side-view endoscopes.

\begin{tabular}{|c|c|c|c|c|}
\hline Type of endoscope & $\begin{array}{l}\text { Sampling from each } \\
\text { endoscope, } n\end{array}$ & $\begin{array}{l}\text { Tip contaminated before } \\
\text { reprocessing, } \mathbf{n}\end{array}$ & $\begin{array}{l}\text { Tips contaminated after } \\
\text { reprocessing, } \mathbf{n}\end{array}$ & $\begin{array}{l}\text { Working channel contaminated } \\
\text { after reprocessing }\end{array}$ \\
\hline Olympus TJF Q 180V) & 67 & $61 / 67$ & $15 / 67$ & $6 / 67$ \\
\hline Olympus TJF $160 \mathrm{R}$ & 35 & $31 / 35$ & $6 / 35$ & $4 / 35$ \\
\hline Total & 102 & $92 / 102$ & $21 / 102$ & $10 / 102$ \\
\hline
\end{tabular}




\begin{tabular}{|c|c|c|c|c|c|c|}
\hline \multirow{3}{*}{$\begin{array}{l}\text { Microorganisms } \\
\text { identified }\end{array}$} & \multirow{2}{*}{\multicolumn{2}{|c|}{$\begin{array}{l}\text { Before reprocessing } \\
\text { Swabs }\end{array}$}} & \multicolumn{4}{|c|}{ After reprocessing } \\
\hline & & & \multicolumn{3}{|l|}{ Swabs } & \multirow{2}{*}{$\begin{array}{l}\text { Normal saline } \\
\text { Sample } \\
\text { Total }\end{array}$} \\
\hline & Direct & Enriched & Direct & Enriched & Total & \\
\hline Streptococcus spp. & 28 & 28 & 1 & - & 1 & - \\
\hline Staphylococcus spp. & 18 & 20 & 1 & 2 & 2 & 2 \\
\hline Escherichia spp. & 31 & 35 & 1 & 1 & 1 & - \\
\hline Klebsiella spp. & 36 & 36 & 2 & 8 & 8 & 1 \\
\hline Serretia spp. & 4 & 6 & - & 3 & 3 & 1 \\
\hline Proteus spp. & 6 & 7 & - & 2 & 2 & - \\
\hline Pseudomonas spp. & 19 & 21 & - & 2 & 2 & 3 \\
\hline Candida spp. & 10 & 6 & - & 4 & 4 & 5 \\
\hline Citrobacter spp. & 6 & 1 & - & - & - & - \\
\hline Yersinia spp. & 8 & 1 & - & - & - & - \\
\hline Enterobacter spp. & 10 & 4 & - & - & - & - \\
\hline Total & 176 & 165 & 5 & 22 & 23 & 12 \\
\hline
\end{tabular}

Table 2 Number of microorganism types identified from the sideview endoscopes before and after manual reprocessing.
(Olympus TJF 160R). Out of the 10 positive liquid samples, 6 were from the Olympus TJF Q $180 \mathrm{~V}$ and 4 from the Olympus TJF $160 \mathrm{R}$ so the culture-positive rates in working channels were $6 / 67$ (9\%) and $4 / 35$ (11\%) for liquid samples taken from the 1-year-old and 5 -year-old scopes, respectively.

Different microbial species were isolated from contaminated tips and working channels after manual reprocessing. The organisms most often isolated from the reprocessed tips were Klebsiella spp. (34\%), Candida spp. (17\%), Serretia spp. (13\%), Pseudomonas spp. (8\%) and Staphylococcus spp.(8\%), while non-albicans Candida spp. (41\%), Pseudomonas spp. (25\%) and Staphylococcus spp. (16\%) were the organisms most often recovered from the reprocessed working channels ( Table 2 ).

After reprocessing, multiple microorganisms were found from contaminated tips after 4/102 sampling procedures (4\%) and from contaminated working channels after 2/102 sampling procedures (2\%). On one occasion Klebsiella spp. was grown four times from the same scope after reprocessing for four times (all these samples were collected and processed on the same day). In yet another occasion Klebsiella spp.and Pseudomonas spp. were isolated from the tip and the working channel of the same side-view endoscope.

\section{Discussion \\ $\nabla$}

Over 400 ERCP procedures are performed annually at the tertiary referral endotherapy unit at the Colombo South Teaching Hospital, Sri Lanka. To minimize the risk of transmission of infections to patients undergoing ERCP, there is strict adherence to the manual reprocessing methods for side-view endoscopes that are recommended by the manufacturer. Procedural errors in cleaning and disinfection of endoscopes have been documented as the cause for failure of reprocessing in other countries even after the instruments have been subjected to full reprocessing cycles [9].

To the best of our knowledge, this is the first study carried out in Sri Lanka to objectively analyze the level of disinfection concerning therapeutic side-view endoscopes. We used environmental culturing of samples from the tips and working channels of sideview endoscopes as surrogate markers to monitor the effectiveness of cleaning and disinfection techniques in a tertiary referral endotherapy unit. Briefly, the manual reprocessing protocol includes pre-cleaning, manual cleaning, high level disinfection
(HLD) using $0.35 \%$ peracetic acid, and rinsing with filtered water after HLD. In this study we have only sampled the working channel and the tip of the endoscope. According to the published literature, other channels including auxiliary water channels and air/water channels can be contaminated during the procedure and reprocessing is recommended [19]. The internal lumen of the TJF 160R model's elevator wire channel is also exposed to contamination. However the published literature shows that the working channels and the tips of endoscopes are the sites most likely to be contaminated during the ERCP procedure [18].

Microbial growth was seen in $20 \%$ of the samples taken from the tip and $9 \%$ of the samples taken from the working channels of reprocessed side-view endoscopes. The isolation rate found in this study was less than that of a previous study done in Korea, in which a $37.2 \%$ culture-positive rate was reported [20]. The majority of bacteria identified from the reprocessed side-view endoscopes in our study were Gram-negative bacilli and the findings are similar to those of a study done by Rerknimitr et al. [21]. Candida spp. was the species most often isolated from the reprocessed working channels in the current study. But Pseudomonas species and Acinetobacter species were the predominant isolates from working channels according to a study by Moses et al. [22]. We identified that Klebsiella spp.as the species most commonly isolated from the tip of the reprocessed side-view endoscopes. Isolation of Klebsiella after reprocessing indicates a risk of patient-to-patient disease transmission; Klebsiella pneumoniae has been identified as a cause for duodenoscope-related nosocomial infection in hospitals in France [23].

The results of our study suggest that disinfection of the tip of the side-view endoscope using manual reprocessing methods was less effective than disinfection of working channels. In $2011 \mathrm{Kim}$ et al. [24] found $4.65 \%$ and $0 \%$ culture-positive rates for the endoscope tip and working channel after automated reprocessing using orthophthalaldehyde disinfectant. This comparison highlights the complexity of the tip of side-view endoscopes with regard to reprocessing, even with automated techniques.

We also observed that in only $80 \%$ of instances were the bacteria isolated after reprocessing the same as those found before reprocessing. This supports the hypothesis that the present protocol prescribed for manual reprocessing of side-view endoscopes is suboptimal. If, on $20 \%$ of occasions the organisms identified after reprocessing had not been present before reprocessing, then they 
may have come from the environment, from handling or from the washing reagent.

Interestingly we have investigated the rinsing solution (filtered water) used for reprocessing; this was found to be sterile on all the occasions. Thus the contamination may have come from the environment or from handling.

Sampling the working channels before reprocessing was not practically possible because of time limitations and the availability of only two working sideview endoscopes. This study was carried out in the only tertiary referral endotherapy unit in Sri Lanka serving for at least 15 patients per session and therefore having an extremely tight schedule between one patient and the next. However we sampled the tip of the endoscope because this did was not take much time.

Reprocessing is a multistep process; involving both humans and chemicals, hence multiple errors are possible [21]. Although traditional reprocessing methods are still used in clinical practice; new technologies with HLD are now widely available [25]. Automated reprocessing is universally recommended by several organizations since it provides a standardized validated reprocessing cycle for clinical settings $[26,27]$. Although there are financial constraints associated with automated reprocessing, the bioburden found in this study would have been significantly less if automated reprocessing had been used rather than manual cleaning. Furthermore, there would have been less human variability and compliance with guidelines would have been better with automated reprocessing.

Disinfection of flexible endoscopes is less effective without adequate manual cleaning because of ineffective contact and penetration of disinfectant in the presence of organic materials [28]. Insufficiently cleaned endoscope channels can promote the formation of microbial plaques and biofilms. Hence, correct manual cleaning is important to remove the organic material which otherwise can fix and promote biofilm formation [17]. Bacterial biofilms contribute to the failure of adequate reprocessing of endoscopes. Biofilms are resistant to most available disinfectants; hence appropriate reprocessing of endoscopes using high level disinfectants is recommended [3]. Moses et al. reported that their culture-positive rate was as high as $14.5 \%$ and they have suggested that faulty mechanical cleaning might have been a possible reason for the failure of the reprocessing procedure [22].

In the endotherapy unit where the present study was carried out, $0.35 \%$ peracetic acid is used as a high level disinfectant for reprocessing. Peracetic acid is a powerful oxidizing agent that rapidly kills a wide range of microorganisms and is active against a broad spectrum of microorganisms including bacteria, viruses, mycobacteria, fungi, yeast, and spores of bacteria [16]. Although the exposure time for peracetic acid is 5 minutes for decontamination of gastrointestinal endoscopes in routine practice, the exposure time for side-view endoscopes has been extended to $10 \mathrm{~min}$ in current practice, to achieve sporicidal activity. Despite the 10minute exposure to disinfectant while reprocessing, a considerable culture-positive rate for reprocessed side-view endoscopes was observed. The effects of peracetic acids on biological deposits (propensity to fix or to remove biofilms from material) have not been completely studied [3].

It is recommended that dedicated work areas should be used for reprocessing to avoid possible cross-contamination [27]. However, in the present study the entire reprocessing procedure was carried out in the same room where ERCP was done. This could further contribute to the high culture-positivity rate.
It was found that $71 \%(15 / 21)$ of the culture-positive samples were taken from the tip of the 1-year-old and more frequently used side-view endoscope (Olympus TJF Q 180V), used 67/102 times. This compared with $29 \%$ (6/21) of the culture-positive samples being taken from the 5-year-old less frequently used endoscope (Olympus TJF 160R), which was used 35/102 times. This observation is also supported by other studies where they have found a higher bioburden associated with the frequent use of endoscopes [29]. In the study only two endoscopes that were currently in use at the endotherapy unit at the tertiary care hospital were studied. The results might have been different if more endoscopes had been included, depending on user frequency of use. Microbiological monitoring can be used to identify deficiencies in reprocessing practices and also to implement corrective actions that will improve future reprocessing efficacy. It was a limitation of this study that viruses and possible uncultivable bacteria were not considered. Thus the real bio burden after reprocessing might have been underestimated. The specimen processing method used in the study would have its own limitations in determining the true bioburden in comparison with the filtration method. However this specimen processing method for detection of the bioburden in endoscopic working channels has been used in several published studies [11].

In conclusion, as high culture-positive rates were observed after reprocessing using the standard manual reprocessing techniques, further investigations with more scopes in different centers on the effectiveness of endoscope reprocessing is needed to rectify reprocessing methods to prevent transmission of infection secondary to ERCP.

\section{Competing interests: None.}

\section{References}

1 Nelson DB, Muscarella LF. Current issues in endoscope reprocessing and infection control during gastrointestinal endoscopy. World J Gastroenterol 2006; 12: 3953-3964

2 Alfa MJ, Olson N, DeGagne P et al. A survey of reprocessing methods, residual viable bioburden, and soil levels in patient-ready endoscopic retrograde cholangiopancreatography duodenoscopes used in Canadian centers. Infect Control Hosp Epedimiol 2002; 23: 198-206

3 Pascu O, Tantau M eds. Therapeutic Gastrointestinal Endoscopy. Croatia: InTech; 2011

4 Chu NS, Favero M. The microbial flora of the gastrointestinal tract and the cleaning of flexible endoscopes. Gastrointest Endosc Clin N Am 2000; 10: 233-244

5 Alfa MJ, Sitter DL. In-hospital evaluation of orthophthalaldehyde as a high level disinfectant for flexible endoscopes. J Hosp Infect 1994; 26: $15-26$

6 Vesley D, Melson J, Stanley P. Microbial bioburden in endoscope reprocessing and an in-use evaluation of the high-level disinfection capabilities of Cidex PA. Gastroenterol Nurs 1999; 22: 63-68

7 Chu NS, McAlister D, Antonoplos PA. Natural bioburden levels detected on flexible gastrointestinal endoscopes after clinical use and manual cleaning. Gastrointest Endosc 1998; 48: 137-142

8 Gastroenterological Nurses College of Australia (GENCA) and Gastroenterological SocietyAustralia (GESA). Infection Control in Endoscopy. 3rd ed. Australia: Gastroenterological SocietyAustralia; 2010

9 Petersen BT, Chennat J, Cohen J et al. Multisociety guideline on reprocessing flexible gastrointestinal endoscopes: 2011. Gastrointest Endosc 2011; 73: 1075-1084

10 Schembre $D B$. Infectious complications associated with gastrointestinal endoscopy. Gastrointest Endosc Clin N Am 2000; 10: 215-232

11 Gillespie EE, Kotsanas D, Stuart RL. Microbiological monitoring of endoscopes: 5-year review. J Gastroenterol Hepatol 2008; 23: 1069-1074

12 Nelson DB, Barkun AN, Block KP et al. Technology status evaluation report. Transmission of infection by gastrointestinal endoscopy. May 2001. . Gastrointest Endosc 2001; 54: 824-828 
13 Ofstead CL, Dirlam LanglayAM, Mueller NJ et al. Re-evaluating endoscopy-associated infection risk estimates and their implications. Am J Infect Control 2013; 41: 734-736

14 Rutala WA, Weber DJ. Reprocessing endoscopes: United States perspective. J Hosp Infect PubMed 2004; 56 (Suppl 02): S27-S39

15 Nelson $D B$. Infectious disease complications of GI endoscopy: part II, exogenous infections. Gastrointest Endosc 2003; 57: 695 - 711

16 Rutala WA, Weber DJ Healthcare Infection Control Practices Advisory Committee (HICPAC). CDC guideline for disinfection and sterilization in healthcare facilities, 2008. Epub 112008

17 Beilenhoff $U$, Neumann CS, Rey JF et al. ESGE-ESGENA guideline for quality assurance in reprocessing: microbiological surveillance testing in endoscopy. Endoscopy 2007; 39: 175-181

18 Catalone B. Microbiological surveillance of flexible endoscopes. Managing Infect Control 2006: 56-60

19 Muscarella LF. Investigation and prevention of infectious outbreaks during endoscopic retrograde cholangiopancreatography. Endoscopy 2010; 42: 957-959

$20 \mathrm{Kim}$ JB, Han DS, Lee HL et al. The value of peracetic Acid (SCOTELIN) for endoscope disinfection. Korean J Gastrointest Endosc 2004; 28: $284-$ 290

21 Rerknimitr R, Eakthunyasakul S, Nunthapisud P et al. Results of gastroscope bacterial decontamination by enzymatic detergent compared to chlorhexidine. World J Gastroenterol 2006; 12: 4199-4202
22 Moses FM, Lee J. Surveillance cultures to monitor quality of gastrointestinal endoscope reprocessing. Am J Gastroenterol 2003; 98: 77-81

23 Aumeran C, Poincloux L, Souweine B et al. Multidrug-resistant Klebsiella pneumoniae outbreak after endoscopic retrograde cholangiopancreatography. Endoscopy 2010; 42: 895-899

24 Kim SY, Lee HS, Hyun JJ et al. Comparison on the efficacy of disinfectants used in automated endoscope reprosessors: PHMB-DBAC versus Orthopthalaldehyde. Clin Endosc 2011; 44: 109-115

25 Desilets D, Kaul V, Tierney WM et al. Automated endoscope reprocessors. Gastrointest Endosc 2010; 72: 675-680

26 Classen M, Tytgat G, Lightdale C. Gastroenterological Endoscopy. Hong Kong: Leo Paper Ltd; 2010

27 Beilenhoff U, Neumann CS, Rey JF et al. ESGE-ESGENA guideline: cleaning and disinfection in gastrointestinal endoscopy. Endoscopy 2008; 40: $939-957$

28 Nelson DB. Recent advances in epidemiology and prevention of gastrointestinal endoscopy related infections. Curr Opin Infect Dis 2005; 18: $326-330$

29 Bisset $L$, Cossart YE, Selby $W$ et al. A prospective study of the efficacy of routine decontamination for gastrointestinal endoscopes and the risk factors for failure. Am J Infect Control 2006; 34: 274-280 